\title{
Multiple Weighted Estimates for Vector-Valued Multilinear Singular Integrals with Non-Smooth Kernels and Its Commutators
}

\author{
Dongxiang Chen, Dan Zou, and Suzhen Mao \\ Department of Mathematics, Jiangxi Normal University, Jiangxi 330022, China \\ Correspondence should be addressed to Dongxiang Chen; chendx020@yahoo.com.cn
}

Received 24 March 2013; Revised 14 September 2013; Accepted 21 September 2013

Academic Editor: L. E. Persson

Copyright (c) 2013 Dongxiang Chen et al. This is an open access article distributed under the Creative Commons Attribution License, which permits unrestricted use, distribution, and reproduction in any medium, provided the original work is properly cited.

This note concerns multiple weighted inequalities for vector-valued multilinear singular integral operator with nonsmooth kernel and its corresponding commutators containing multilinear commutator and iterated commutator generated by the vector-valued multilinear operator and BMO functions. By the weighted estimates for a class of new variant maximal and sharp maximal functions, the multiple weighted norm inequalities for such operators are obtained.

\section{Introduction}

It is well known that multiple weighted norm inequalities for multilinear operators and their related commutators on various spaces of function is a center topic of harmonic analysis, which recently attracts a lot of attention, see [1-3] et al.

In this paper, we will focus on the multiple weighted estimates for vector valued multilinear singular integral with nonsmooth kernel and its commutators. Now we give some information on multilinear Calderón-Zygmund operators.

The multilinear operator $T$ we study is initially defined on the $m$-fold product of Schwartz space $\mathcal{S}\left(\mathbb{R}^{n}\right) \times \cdots \times \mathcal{S}\left(\mathbb{R}^{n}\right)$ and taking values into the space of tempered distributions $\mathcal{S}^{\prime}\left(\mathbb{R}^{n}\right)$; that is,

$$
T: \mathcal{S}\left(\mathbb{R}^{n}\right) \times \cdots \times \mathcal{S}\left(\mathbb{R}^{n}\right) \longrightarrow \mathcal{S}^{\prime}\left(\mathbb{R}^{n}\right) .
$$

A locally integrable function $K\left(x, y_{1}, \ldots, y_{m}\right)$ defined away from the diagonal $x=y_{1}=\cdots=y_{m}$ in $\left(\mathbb{R}^{n}\right)^{m+1}$ is called an associated kernel of $T$ if

$$
\begin{aligned}
T & \left(f_{1}, \ldots, f_{m}\right)(x) \\
\quad & =\int_{\left(\mathbb{R}^{n}\right)^{m}} K\left(x, y_{1}, \ldots, y_{m}\right) f_{1}\left(y_{1}\right) \cdots f_{m}\left(y_{m}\right) d y_{1} \cdots d y_{m},
\end{aligned}
$$

where $f_{1}, \ldots, f_{m}$ are $C^{\infty}$ functions with compact support and for all $x \notin \bigcap_{j=1}^{m} \operatorname{supp} f_{j}$.

Moreover, we assume the associated kernel $K$ satisfies the following standard estimates:

$$
\left|K\left(y_{0}, y_{1}, \ldots, y_{m}\right)\right| \leq \frac{A}{\left(\sum_{k, l=0}^{m}\left|y_{k}-y_{l}\right|\right)^{m n}},
$$

for some $A>0$ and all $\left(y_{0}, \ldots, y_{m}\right)$ with $y_{0} \neq y_{j}$ for some $j \in\{1, \ldots, m\}$, and

$$
\begin{aligned}
& \left|K\left(y_{0}, \ldots, y_{j}, \ldots, y_{m}\right)-K\left(y_{0}, \ldots, y_{j}^{\prime}, \ldots, y_{m}\right)\right| \\
& \quad \leq \frac{A\left|y_{j}-y_{j}^{\prime}\right|^{\varepsilon}}{\left(\sum_{k, l=0}^{m}\left|y_{k}-y_{l}\right|\right)^{m n+\varepsilon}},
\end{aligned}
$$

for some $\varepsilon>0$ and all $0 \leq j \leq m$, where $\left|y_{j}-y_{j}^{\prime}\right| \leq(1 / 2)$ $\max _{0 \leq k \leq m}\left|y_{j}-y_{k}\right|$. These facts can be founded in [4].

We now turn to present the definitions of $m$-linear commutator and iterated commutator of multilinear singular integral. 
For the multilinear operator $T$ and $\vec{b}=\left(b_{1}, \ldots, b_{m}\right)$ in $\mathrm{BMO}^{m}$, we define the $m$-linear commutator $T_{\Sigma \vec{b}}$ as the following form:

$$
T_{\Sigma \vec{b}}(\vec{f})(x)=\sum_{j=1}^{m} T_{b_{j}}^{j}(\vec{f})(x):=\sum_{j=1}^{m}\left[b_{j}, T\right]_{j}\left(f_{1}, \ldots, f_{m}\right)(x),
$$

where

$$
\begin{aligned}
T_{b_{j}}^{j}(\vec{f})(x)= & b_{j}(x) T\left(f_{1}, \ldots, f_{m}\right)(x) \\
& -T\left(f_{1}, \ldots, b_{j} f_{j}, \ldots, f_{m}\right)(x) . \\
T_{\Pi \vec{b}}(\vec{f})(x):= & {\left[b_{1},\left[b_{2}, \ldots\left[b_{m-1},\left[b_{m}, T\right]_{m}\right]_{m-1}, \ldots\right]_{2}\right]_{1} } \\
& \times\left(f_{1}, \ldots, f_{m}\right)(x) .
\end{aligned}
$$

If $T$ is associated with a distribution kernel, which coincides with the function $K$ defined away from the diagonal $x=y_{1}=$ $\cdots=y_{m}$ in $\left(\mathbb{R}^{n}\right)^{m+1}$, then

$$
\begin{aligned}
& T_{b_{j}}^{j}(\vec{f})(x) \\
& \quad=\int_{\left(\mathbb{R}^{n}\right)^{m}} K\left(x, y_{1}, \ldots, y_{m}\right) f_{1}\left(y_{1}\right) \cdots \\
& \quad\left(b_{j}(x)-b_{j}\left(y_{j}\right)\right) f_{j}\left(y_{j}\right) \cdots f_{m}\left(y_{m}\right) d y_{1} \cdots d y_{m} .
\end{aligned}
$$

And we also present the iterated commutator $T_{\Pi \vec{b}}$,

$$
\begin{aligned}
T_{\Pi \vec{b}}(\vec{f})(x)= & \int_{\left(\mathbb{R}^{n}\right)^{m}} K\left(x, y_{1}, \ldots, y_{m}\right) \\
& \quad \times \prod_{j=1}^{m}\left(b_{j}(x)-b_{j}\left(y_{j}\right)\right) f_{j}\left(y_{j}\right) d y_{1} \cdots d y_{m} .
\end{aligned}
$$

Here the notations of commutators are taken from $[5,6]$.

The following class of weights were introduced in [1]. Let $1 \leq p_{1}, \ldots, p_{m}<\infty$, and $1 / m \leq p<\infty$, with $1 / p=1 / p_{1}+$ $\cdots+1 / p_{m}$, and $\vec{P}=\left(p_{1}, \ldots, p_{m}\right)$; given $\vec{\omega}=\left(\omega_{1}, \ldots, \omega_{m}\right)$, we say that $\vec{\omega}$ satisfies the $A_{\vec{p}}$ condition if

$$
\sup _{Q}\left(\frac{1}{|Q|} \int_{Q} \nu_{\vec{\omega}}\right)^{1 / p} \prod_{j=1}^{m}\left(\frac{1}{|Q|} \int_{Q} \omega_{j}^{1-p_{j}^{\prime}}\right)^{1 / p_{j}^{\prime}}<\infty
$$

where $v_{\vec{\omega}}=\prod_{j=1}^{m} \omega_{j}^{p / p_{j}}$, when $p_{j}=1, \quad\left((1 /|Q|) \int_{Q} \omega_{j}^{1-p_{j}^{\prime}}\right)^{1 / p_{j}^{\prime}}$ is understood as $\left(\inf _{Q} \omega_{j}\right)^{-1}$.

Observe that $A_{(1, \ldots, 1)}$ is contained in $A_{\vec{P}}$ for each $\vec{P}$. However, the class $A_{\vec{P}}$ is not increasing with the natural partial order, see [1] for detail. Lerner et al. [1] established multiple weighted estimates for multilinear $\mathrm{C}-\mathrm{Z}$ operators and that for $m$-linear commutator of multilinear $C-Z$ commutator. In 2012, Chen and $\mathrm{Wu}$ [2] extend their results to $m$-linear commutator and iterated commutator of multilinear $\mathrm{C}-\mathrm{Z}$ operator with nonsmooth kernel satisfying Assumptions (H1) and (H2).

Next we define the vector-valued multilinear operator $T_{q}(q>0)$ associated with the operator $T$ by

$$
\begin{aligned}
T_{q}\left(f_{1}, \ldots, f_{m}\right)(x) & =\left|T\left(f_{1}, \ldots, f_{m}\right)\right|_{q}(x) \\
& =\left(\sum_{k=1}^{\infty}\left|T\left(f_{1 k}, \ldots, f_{m k}\right)(x)\right|^{q}\right)^{1 / q},
\end{aligned}
$$

where $f_{i}=\left\{f_{i k}\right\}_{k=1}^{\infty}$, for $i=1, \ldots, m$.

This operator was first studied by Grafakos and Martell in [7]. Later Cruz-Uribe et al. gained the weak boundedness of this one in [8]. We list them as follows.

Theorem A (see [7]). Let $T$ be a multilinear CalderónZygmund operator as before, and let $1 / m<p<\infty, 1 / p=$ $1 / p_{1}+\cdots+1 / p_{m}$ with $1<p_{1}, \ldots, p_{m}<\infty, 1 / m<q<\infty$, and $1 / q=1 / q_{1}+\cdots+1 / q_{m}$ with $1<q_{1}, \ldots, q_{m}<\infty$. There exists a constant $C>0$ such that

$$
\left\|T_{q}(\vec{f})\right\|_{L^{p}\left(\mathbb{R}^{n}\right)} \leq C \prod_{j=1}^{m}\left\|\left|f_{j}\right|_{q_{j}}\right\|_{L^{p_{j}\left(\mathbb{R}^{n}\right)}} .
$$

For the sequence $\left\{\vec{f}_{k}\right\}_{k=1}^{\infty}=\left(f_{1 k}, \ldots, f_{m k}\right)_{k=1}^{\infty}$, the vectorvalued version of the commutators $T_{\Sigma \vec{b}, q}$ and $T_{\Pi \vec{b}, q}$ are given by

$$
\begin{aligned}
T_{\Sigma \vec{b}, q}(\vec{f})(x) & =\left|T_{\Sigma \vec{b}}(\vec{f})\right|_{q}(x) \\
& =\left(\sum_{k=1}^{\infty}\left|T_{\Sigma \vec{b}}\left(f_{1 k}, \ldots, f_{m k}\right)(x)\right|^{q}\right)^{1 / q}, \\
T_{\Pi \vec{b}, q}(\vec{f})(x) & =\left|T_{\Pi \vec{b}}(\vec{f})\right|_{q}(x) \\
& =\left(\sum_{k=1}^{\infty}\left|T_{\Pi \vec{b}}\left(f_{1 k}, \ldots, f_{m k}\right)(x)\right|^{q}\right)^{1 / q} .
\end{aligned}
$$

In 2008, Tang established weighed norm inequalities for the commutators of vector-valued multilinear operator in [6], but their results are not the multiple weighted estimates that are obtained by Lerner et al. in [1].

Now we restore to give some information on the kernel $K$ which satisfy Assumptions (H1) and (H2). Let $\left\{A_{t}\right\}_{t>0}$ be a class of integral operators which play the role of the approximation to the identity. We always assume that the operators $A_{t}$ are associated with kernels $a_{t}(x, y)$ in the sense that for all $f \in \bigcup_{p \in[1, \infty]} L^{p}$ and $x \in \mathbb{R}^{n}$

$$
A_{t} f(x)=\int_{\mathbb{R}^{n}} a_{t}(x, y) f(y) d y,
$$

and that the kernels $a_{t}(x, y)$ satisfy the following conditions

$$
\left|a_{t}(x, y)\right| \leq h_{t}(x, y):=t^{-n / s} h\left(\frac{|x-y|}{t^{1 / s}}\right)
$$


where $s$ is a positive fixed constant and $h$ is a positive, bounded, decreasing function satisfying that for some $\eta>0$,

$$
\lim _{r \rightarrow \infty} r^{n+\eta} h\left(r^{s}\right)=0
$$

Recall that the $j$ th transpose $T^{*, j}$ of the $m$-linear operator $T$ is defined via

$$
\begin{aligned}
\left\langle T^{*, j}\right. & \left.\left(f_{1}, \ldots, f_{m}\right), g\right\rangle \\
& =\left\langle T\left(f_{1}, \ldots, f_{j-1}, g, f_{j+1}, \ldots, f_{m}\right), f_{j}\right\rangle,
\end{aligned}
$$

for all $f_{1}, \ldots, f_{m}, g$ in $\delta\left(\mathbb{R}^{n}\right)$. Notice that the kernel $K^{*, j}$ of $T^{*, j}$ is related to the kernel $K$ of $T$ via the identity

$$
K^{*, j}\left(x, j_{1}, \ldots, y_{j}, \ldots, y_{m}\right)=K\left(y_{j}, y_{1}, \ldots, x, \ldots, y_{m}\right) .
$$

If an $m$-linear operator $T$ maps a product of Banach Spaces $X_{m} \times \cdots \times X_{m}$ to another Banach Space $X$; then transpose $T^{*, j}$ maps the product of Banach Spaces $X_{1} \times \cdots \times$ $X_{j-1} \times X_{j}^{*} \times X_{j+1} \times \cdots \times X_{m}$ into $X_{j}^{*}$. Moreover, the norms of $T$ and $T^{*, j}$ are equal. To maintain uniform notation, we may occasionally denote $T$ by $T^{*, 0}$ and $K$ by $K^{*, 0}$.

Assumption (H1). Assume that for each $i=1, \ldots, m$, there exist operators $\left\{A_{t}^{i}\right\}_{t>0}$ with kernel $a_{t}^{i}(x, y)$ satisfying conditions $s$ and $\eta$ and that for every $j=0,1, \ldots, m$, there exist kernels $K_{t}^{*, j(i)}\left(x, y_{1}, \ldots, y_{m}\right)$ such that

$$
\begin{gathered}
\left\langle T^{*, j}\left(f_{1}, \ldots, A_{t}^{(i)} f_{j}, \ldots, f_{m}\right), g\right\rangle \\
=\int_{\mathbb{R}^{n}} \int_{\left(\mathbb{R}^{n}\right)^{m}} K_{t}^{*, j(i)}\left(x, y_{1}, \ldots, y_{m}\right) f_{1}\left(y_{1}\right) \ldots \\
f_{m}\left(y_{m}\right) g(x) d \vec{y} d x
\end{gathered}
$$

for all $f_{1}, \ldots, f_{m}$ in $\mathcal{S}\left(\mathbb{R}^{n}\right)$ with $\bigcap_{k=1}^{m} \operatorname{supp} f_{k} \cap \operatorname{supp} g=\emptyset$. Also assume that for every $j=0,1, \ldots, m$ and every $i=$ $1, \ldots, m$, we have

$$
\begin{aligned}
\left|K^{*, j}(x, \vec{y})-K_{t}^{*, j(i)}(x, \vec{y})\right| & \leq \frac{A}{\left(\sum_{k=1}^{m}\left|x-y_{k}\right|\right)^{m n}} \\
& \times \sum_{k=1, k \neq i}^{m} \Phi\left(\frac{\left|y_{i}-y_{k}\right|}{t^{1 / s}}\right) \\
& +\frac{A t^{\epsilon / s}}{\left(\sum_{k=1}^{m}\left|x-y_{k}\right|\right)^{m n+\epsilon}},
\end{aligned}
$$

where $t^{1 / s} \leq\left|x-y_{i}\right| / 2, \vec{y}=\left(y_{1}, \ldots, y_{m}\right)$.

If $T$ satisfies Assumption (H1), we will say that $T$ is an $m$-linear operator with generalized CaderónZygmund kernel $K$. The collection of function $K$ satisfying (19) and (20) with parameters $m, A, s, \eta$ and $\epsilon$ will be denoted by $m-\operatorname{GCZK}(A, s, \eta, \epsilon)$, we say that $T$ is of class
$m-\operatorname{GCZK}(A, s, \eta, \epsilon)$ if $T$ has an associated kernel $K$ in $m-\operatorname{GCZK}(A, s, \eta, \epsilon)$.

Assumption (H2). Assume that there exist operators $\left\{B_{t}\right\}_{t>0}$ with kernel $b_{t}(x, y)$ satisfying conditions (15) and (16) with constants $s$ and $\eta$. Let

$$
K_{t}^{(0)}\left(x, y_{1}, \ldots, y_{m}\right)=\int_{\mathbb{R}^{n}} K\left(z, y_{1}, \ldots, y_{m}\right) b_{t}(x, z) d z,
$$

whenever $2 t^{1 / s} \leq \min _{1 \leq j \leq m}\left|x-y_{j}\right|$ and

$$
\begin{aligned}
\left|K(x, \vec{y})-K_{t}^{(0)}\left(x^{\prime}, \vec{y}\right)\right| \leq & \frac{A}{\left(\sum_{k=1}^{m}\left|x-y_{k}\right|\right)^{m n}} \\
& \times \sum_{k=1, k \neq i}^{m} \Phi\left(\frac{\left|y_{i}-y_{k}\right|}{t^{1 / s}}\right) \\
& +\frac{A t^{\epsilon / s}}{\left(\sum_{k=1}^{m}\left|x-y_{k}\right|\right)^{m n+\epsilon}}
\end{aligned}
$$

whenever $2\left|x-x^{\prime}\right| \leq t^{1 / s}$ and $2 t^{1 / s} \leq \max _{1 \leq j \leq m}\left|x-y_{j}\right|$.

When $T$ is of $m-\operatorname{GCZK}(A, s, \eta, \epsilon)$ and its kernel also satisfies Assumption (H2), Duong et al. in [5] proved that multilinear singular integral $T$ is bounded from $L^{p_{1}}\left(\mathbb{R}^{n}\right) \times$ $\cdots \times L^{p_{m}}\left(\mathbb{R}^{n}\right)$ to $L^{p}\left(\mathbb{R}^{n}\right)\left(L^{p, \infty}\left(\mathbb{R}^{n}\right)\right)$ for $0<p<\infty, 1 \leq$ $p_{1}, \ldots, p_{m}<\infty$ with $1 / p=1 / p_{1}+\cdots+1 / p_{m}$. And they also remarked that the above kernel which they studied has weaker regularity. It is natural to ask whether the vectorvalued multilinear operator $T_{q}$ with kernels satisfying the same conditions as in [5] and its commutators $T_{\Sigma \vec{b}, q}$ and $T_{\Pi \vec{b}, q}$ have multiple weighted estimates or not. These problems will be addressed by our next theorems.

Now we can formulate our results as follows.

Theorem 1. Assume that $T_{q}$ is a vector-valued multilinear operator defined as (10) associated with $T$ being an $m-G C Z K(A, s, \eta, \epsilon)$ whose kernel K satisfies Assumption (H2). If there exist $1 \leq p_{1}, \ldots, p_{m}<\infty$, with $1 / p=1 / p_{1}+\cdots+1 / p_{m}$ and $\vec{\omega}=\left(\omega_{1}, \ldots, \omega_{m}\right) \in A_{\vec{P}}$ with $\vec{P}=\left(p_{1}, \ldots, p_{m}\right)$ then

(i) $T_{q}$ can be extended to a bounded operator from $L^{p_{1}}\left(\omega_{1}\right) \times \cdots \times L^{p_{m}}\left(\omega_{m}\right)$ to $L^{p}\left(\nu_{\vec{\omega}}\right)$ if all exponents $p_{j}$ are strictly greater than 1 ;

(ii) $T_{q}$ can be extended to a bounded operator from $L^{p_{1}}\left(\omega_{1}\right) \times \cdots \times L^{p_{m}}\left(\omega_{m}\right)$ to $L^{p, \infty}\left(\nu_{\vec{\omega}}\right)$ if $1 \leq p_{j}<\infty$, $j=1, \ldots, m$, and at least one of the $p_{j}=1$.

Theorem 2. Assume that $T_{q}$ is a vector-valued multilinear operator that satisfies the assumptions in Theorem 1, and the multilinear commutator $T_{\Sigma \vec{b}, q}$ is defined as (12). Let $\vec{b} \in B M O^{m}$, $1<p_{1}, \ldots, p_{m}<\infty, 1<p<\infty$ with $1 / p=1 / p_{1}+\cdots+1 / p_{m}$, and $\vec{\omega}=\left(\omega_{1}, \ldots, \omega_{m}\right) \in A_{\vec{P}}$ with $\vec{P}=\left(p_{1}, \ldots, p_{m}\right)$. Then there exists a constant $C>0$ such that

$$
\left\|T_{\Sigma \vec{b}, q}(\vec{f})\right\|_{L^{p}\left(v_{\vec{\omega}}\right)} \leq C \sum_{i=1}^{m}\left\|b_{i}\right\|_{B M O} \prod_{j=1}^{m}\left\|\left|f_{j}\right|_{q_{j}}\right\|_{L^{p_{j}}\left(\omega_{j}\right)} .
$$


Theorem 3. Assume that $T_{q}$ is a vector-valued multilinear operator that satisfies the assumptions in Theorem 1, and the iterated commutator $T_{\Pi \vec{b}, q}$ is defined as (13). Let $\vec{b} \in B M O^{m}$, $1<p_{1}, \ldots, p_{m}<\infty, 1<p<\infty$ with $1 / p=1 / p_{1}+\cdots+$ $1 / p_{m} \vec{\omega}=\left(\omega_{1}, \ldots, \omega_{m}\right) \in A_{\vec{P}}$ with $\vec{P}=\left(p_{1}, \ldots, p_{m}\right)$. Then there exists a constant $C>0$ such that

$$
\left\|T_{\Pi \vec{b}, q}(\vec{f})\right\|_{L^{p}\left(v_{\vec{\omega}}\right)} \leq C \prod_{i=1}^{m}\left\|b_{i}\right\|_{B M O} \prod_{j=1}^{m}\left\|\left|f_{j}\right|_{q_{j}}\right\|_{L^{p_{j}}\left(\omega_{j}\right)},
$$

where $\nu_{\vec{\omega}}=\prod_{j=1}^{m} \omega_{j}^{p / p_{j}}, 1<q_{1}, \ldots, q_{m}<\infty, 1 / m<q<\infty$, and $1 / q=1 / q_{1}+\cdots+1 / q_{m}$.

The rest of this paper is organized as follows. In Section 2, we recall some standard definitions and lemmas. In Section 3, we introduce a class of new maximal functions and prove some useful estimates which will play key roles in the proofs of our theorems. In Section 4, it is devoted to the proof our theorems. Throughout this paper, we use the letter $C$ to denote a positive constant that varies from line to line, but it is independent of the essential variable.

\section{Some Preliminaries}

Lemma 4 (see $\left[1\right.$, Lemma 6.1]). Assume that $\vec{\omega}=\left(\omega_{1}, \ldots, \omega_{m}\right)$ satisfies $A_{\vec{P}}$ condition, then there exists a finite constant $r>1$ such that $\vec{\omega} \in A_{\vec{P} / r}$.

We recall the definition of the Hardy-Littlewood maximal function and the sharp maximal function:

$$
\begin{aligned}
M(f)(x) & =\sup _{Q \ni x} \frac{1}{|Q|} \int_{Q}|f(y)| d y, \\
M^{\sharp}(f)(x) & =\sup _{Q \ni x} \inf _{x} \frac{1}{|Q|} \int_{Q}|f(y)-c| d y \\
& \approx \sup _{Q \ni x} \frac{1}{|Q|} \int_{Q}\left|f(y)-f_{Q}\right| d y,
\end{aligned}
$$

and their variants $M_{\delta}(f)(x)=M\left(|f|^{\delta}\right)^{1 / \delta}(x)$ and $M_{\delta}^{\sharp}(f)(x)$ $=M^{\sharp}\left(|f|^{\delta}\right)^{1 / \delta}(x)$.

We will use the following inequality (see [9]):

$$
\int_{\mathbb{R}^{n}}\left(M_{\delta}(f)(x)\right)^{p} \omega(x) d x \leq C \int_{\mathbb{R}^{n}}\left(M_{\delta}^{\sharp}(f)(x)\right)^{p} \omega(x) d x,
$$

all functions $f$ for which the left-hand side is finite, and where $0<p, \delta<\infty, \omega \in A_{\infty}$.

Now we introduce some facts on Orlicz spaces. Let $\Phi$ : $[0, \infty) \rightarrow[0, \infty)$ be a Young function, that is, a continuous, convex, increasing function with $\Phi(0)=0$ and such that $\Phi(t) \rightarrow \infty$ as $t \rightarrow \infty$. In this paper, any Young function $\Phi$ will be doubling, namely $\Phi(2 t) \leq C \Phi(t)$ for $t>0$. We define the $\Phi$-average of function $f$ over a cube $Q$ by

$$
\|f\|_{\Phi, Q}=\inf \left\{\lambda>0: \frac{1}{|Q|} \int_{Q} \Phi\left(\frac{|f(x)|}{\lambda}\right) d x \leq 1\right\} .
$$

It is a simple but important observation that

$$
\|f\|_{\Phi, Q}>1 \text { iff } \frac{1}{|Q|} \int_{Q} \Phi\left(\frac{|f(x)|}{\lambda}\right) d x>1 .
$$

A particular case of interest, and especially in this paper, is the Young function $\Phi(t)=t\left(1+\log ^{+} t\right)$, the average $\|f\|_{\Phi, Q}$ of a function $f$ given by the Luxemburg norm $\|f\|_{L(\log L), Q}$.

Associated with this average, we have a maximal function

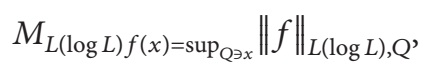

where the supremum is taken over all the cubes containing $x$.

By the generalized Hölder's inequality, we also get

$$
\frac{1}{|Q|} \int_{Q}|f(x) g(x)| d x \leq C\|f\|_{\mathrm{BMO}}\|g\|_{L(\log L), Q} .
$$

\section{New Maximal Functions}

In this section, we will introduce certain variant multilinear maximal functions and establish the multiple weighted estimates for such functions, which are one of the main parts in this paper.

Recall the definitions of these maximal functions, which are introduced by Lerner et al. in [1]

$$
\begin{gathered}
\mathscr{M}(\vec{f})(x)=\sup _{\mathrm{Q} \ni x} \prod_{j=1}^{m} \frac{1}{|Q|} \int_{Q}\left|f_{j}(y)\right| d y, \\
\mathscr{M}_{r}(\vec{f})(x)=\sup _{\mathrm{Q} \ni x} \prod_{j=1}^{m}\left(\frac{1}{|Q|} \int_{Q}\left|f_{j}(y)\right|^{r} d y\right)^{1 / r}, \\
\mathscr{M}_{L(\log L)}(\vec{f})(x)=\sup _{\mathrm{Q} \ni x} \prod_{j=1}^{m}\left\|f_{j}\right\|_{L(\log L), Q^{*}}
\end{gathered}
$$

The fact that $r>1$, there exists a constant $C>0$ such that

$$
\|f\|_{L(\log L), Q} \leq C\left(\frac{1}{|Q|} \int_{Q}\left|f_{j}(y)\right|^{r} d y\right)^{1 / r}
$$

so it is easy to check that

$$
\mathscr{M}(\vec{f})(x) \leq \mathscr{M}_{L(\log L)}(\vec{f})(x) \leq \mathscr{M}_{r}(\vec{f})(x) .
$$

Characterizations of the multiple weights in terms of $\mathscr{M}$ were proven in Theorems 3.3 and 3.7 in [1].

Lemma 5. Let $1 \leq p_{1}, \ldots, p_{m}<\infty, 1 / p=1 / p_{1}+\cdots+1 / p_{m}$, and $\vec{P}=\left(p_{1}, \ldots, p_{m}\right)$,

(i) If $1<p_{1}, \ldots, p_{m}<\infty$, then $\mathscr{M}$ is bounded from $L^{p_{1}}\left(\omega_{1}\right) \times \cdots \times L^{p_{m}}\left(\omega_{m}\right)$ to $L^{p}\left(\nu_{\vec{\omega}}\right)$ if and only if $\vec{\omega}=$ $\left(\omega_{1}, \ldots, \omega_{m}\right) \in A_{\vec{P}}$

(ii) If $1 \leq p_{1}, \ldots, p_{m}<\infty$, then $\mathscr{M}$ is bounded from $L^{p_{1}}\left(\omega_{1}\right) \times \cdots \times L^{p_{m}}\left(\omega_{m}\right)$ to $L^{p, \infty}\left(\nu_{\vec{\omega}}\right)$ if and only if $\vec{\omega}=\left(\omega_{1}, \ldots, \omega_{m}\right) \in A_{\vec{p}}$. 
In the following, we introduce the modified multilinear maximal functions.

Let $r>1,1 \leq l<m, \varrho=\left\{j_{1}, \ldots, j_{l}\right\} \subseteq\{1, \ldots, m\}$ and $\varrho^{\prime}=$ $\{1, \ldots, m\} \backslash \varrho$. We defined the following multilinear maximal functions:

$$
\begin{aligned}
& \mathscr{M}_{Q}\left(|\vec{f}|_{q}\right)(x) \\
& :=\sup _{Q \ni x} \sum_{\nu=0}^{\infty} 2^{-\nu n l} \prod_{j \in \varrho} \frac{1}{|Q|} \int_{Q}\left|f_{j}\right|_{q_{j}} d y_{j} \\
& \times \prod_{j \in \varrho^{\prime}} \frac{1}{\left|2^{v} \mathrm{Q}\right|} \int_{2^{v} \mathrm{Q}}\left|f_{j}\right|_{q_{j}} d y_{j}, \\
& \mathscr{M}_{Q, L(\log L)}\left(|\vec{f}|_{q}\right)(x) \\
& :=\sup _{Q \ni x} \sum_{\nu=0}^{\infty} 2^{-\nu n l} \prod_{j \in \varrho}\left\|\left.|| f_{j}\right|_{q_{j}}\right\|_{L(\log L), Q} \\
& \times \prod_{j \in \varrho^{\prime}}\left\|\left|f_{j}\right|_{q_{j}}\right\|_{L(\log L), 2^{v} Q}, \\
& \mathscr{M}_{Q, r}\left(|\vec{f}|_{q}\right)(x) \\
& :=\sup _{Q \ni x} \sum_{\nu=0}^{\infty} 2^{-v n l} \prod_{j \in \varrho}\left(\frac{1}{|Q|} \int_{Q}\left|f_{j}\right|_{q_{j}}^{r} d y_{j}\right)^{1 / r} \\
& \times \prod_{j \in Q^{\prime}}\left(\frac{1}{\left|2^{v} \mathrm{Q}\right|} \int_{2^{v} \mathrm{Q}}\left|f_{j}\right|_{q_{j}}^{r} d y_{j}\right)^{1 / r},
\end{aligned}
$$

where $\left|f_{j}\right|_{q_{j}}=\left(\sum_{k=1}^{\infty}\left|f_{j k}\left(y_{j}\right)\right|^{q_{j}}\right)^{1 / q_{j}}$.

We remark that when $\varrho=1, \ldots, l$ and $\vec{f}=\left(f_{1}, \ldots, f_{m}\right)$, $\mathscr{M}_{\rho}$ was first introduced by Grafakos el al. in [10] and denoted $\mathscr{M}_{Q}$ by $\mathscr{M}_{l}$. Chen and Wu [2] proved the multiple weighted norm inequality for $\mathscr{M}_{Q, r}$. Similarly to (33), for any $r>0$, we have

$$
\mathscr{M}_{\varrho}\left(|\vec{f}|_{q}\right) \leq \mathscr{M}_{\varrho, L(\log L)}\left(|\vec{f}|_{q}\right) \leq \mathscr{M}_{\varrho, r}\left(|\vec{f}|_{q}\right)
$$

Lemma 6. Let $1<p_{1}, \ldots, p_{m}<\infty, 1 / p=1 / p_{1}+\cdots+1 / p_{m}$, $\vec{P}=\left(p_{1}, \ldots, p_{m}\right), \vec{\omega} \in A_{\vec{p}}$, and $\varrho=\left\{j_{1}, \ldots, j_{l}\right\} \subseteq\{1, \ldots, m\}$, $1 \leq l<m$. Then for some $r>1$ ( $r$ depending only on $\vec{\omega})$, $\mathscr{M}_{r}$ and $\mathscr{M}_{Q, r}$ defined by (36) are bounded from $L^{p_{1}}\left(\omega_{1}\right) \times \cdots \times$ $L^{p_{m}}\left(\omega_{m}\right)$ to $L^{p}\left(\nu_{\vec{\omega}}\right)$. $\mathscr{M}, \mathscr{M}_{L(\log L)}, \mathscr{M}_{\varrho}$, and $\mathscr{M}_{Q, r}$ are bounded from $L^{p_{1}}\left(\omega_{1}\right) \times \cdots \times L^{p_{m}}\left(\omega_{m}\right)$ to $L^{p}\left(\nu_{\vec{\omega}}\right)$.

\section{Weighted Inequalities for Vector-Valued Singular Integral and Its Commutators}

To prove our theorems, we first give two Lemmas about vector-valued operator $T_{q}$ associated with $T$ in $m-\operatorname{GCZO}(A, s, \eta, \epsilon)$ which were obtained by Duong et al. in [5].

Lemma 7. Let $T$ be a multilinear operator in $m-G C Z O$ $(A, s, \eta, \epsilon)$ with $K$ satisfying Assumption (H2). And let $1 / m<$ $p<\infty, 1<p_{1}, \ldots, p_{m}<\infty$ with $1 / p=1 / p_{1}+\cdots+$ $1 / p_{m}, 1 / m<q<\infty$, and $1<q_{1}, \ldots, q_{m}<\infty$ with $1 / q=1 / q_{1}+\cdots+1 / q_{m}$. Then there exists a constant $C>0$ such that

$$
\|T(\vec{f})\|_{L^{p}\left(\mathbb{R}^{n}\right)} \leq C \prod_{j=1}^{m}\|f\|_{L^{p_{j}}\left(\mathbb{R}^{n}\right)} .
$$

Lemma 8. Let $T$ be a multilinear operator in $m$-GCZO $(A, s, \eta, \epsilon)$ with $K$ satisfying Assumption (H2). And let $1 / m<$ $p<\infty, 1 \leq p_{1}, \ldots, p_{m}<\infty$ with $1 / p=1 / p_{1}+\cdots+1 / p_{m}$, and $1 / m<q<\infty, 1<q_{1}, \ldots, q_{m}<\infty$ with $1 / q=$ $1 / q_{1}+\cdots+1 / q_{m}$. Then there exists a constant $C>0$ such that

$$
\|T(\vec{f})\|_{L^{p, \infty}\left(\mathbb{R}^{n}\right)} \leq C \prod_{j=1}^{m}\|f\|_{L^{p_{j}\left(\mathbb{R}^{n}\right)}} .
$$

Before proving Theorem 1, we first present the estimates on the pointwise estimates for sharp Fefferman-stein maximal function action on $T_{q}$.

Proposition 9. Let $T$ be an m-linear operator in GCZO $(A, s, \eta, \epsilon)$ and $T_{q}$ satisfy the assumption in Theorem 1 . Assume that $\varrho_{0}=\{1, \ldots, m\}$ and $0<\delta<1 / m$. For any $\left\{f_{1 m}, \ldots\right.$, $\left.f_{m k}\right\}_{k=1}^{\infty}$ in the product spaces $L^{p_{1}}\left(l^{q_{1}}\right) \times \cdots \times L^{p_{m}}\left(l^{q_{m}}\right)$ with $1<p_{j}, q_{j}<\infty$ for $j=1, \ldots, m$, then these exists a constant $C>0$ such that

$$
M_{\delta}^{\sharp}\left(T_{q}(\vec{f})\right)(x) \leq C\left(\mathscr{M}\left(|\vec{f}|_{q}\right)(x)+\mathscr{M}_{Q}\left(|\vec{f}|_{q}\right)(x)\right) .
$$

The ideas and arguments used in the proof are similar to those in [1] with some modifications. For completeness, we give the proof as follows.

Proof of Proposition 9. For a point $x$ and a cube $Q \ni x$, since $\left.|| \alpha\right|^{r}-|\beta|^{r}|\leq| \alpha-\left.\beta\right|^{\beta}$, for $0<r<1$, to obtain (40), it suffices to prove for $0<\delta<1 / m$

$$
\begin{aligned}
& \left(\frac{1}{|Q|} \int_{Q}\left|T_{q}(\vec{f})(z)-C_{Q}\right|^{\delta} d z\right)^{1 / \delta} \\
& \quad \leq C\left(\mathscr{M}\left(|\vec{f}|_{q}\right)(x)+\mathscr{M}_{Q}\left(|\vec{f}|_{q}\right)(x)\right),
\end{aligned}
$$

for some constant $C_{Q}$ to be determined later.

Let $\left\{f_{1 k}, \ldots, f_{m k}\right\}_{k=1}^{\infty}$ be any smooth vector-valued functions. Set each $\vec{f}_{k}=\vec{f}_{k}^{0}+\vec{f}_{k}^{\infty}$, where $\left\{\vec{f}_{k}^{0}\right\}_{k=1}^{\infty}=\left\{\vec{f}_{k} \chi_{\mathrm{Q}^{*}}\right\}_{k=1}^{\infty}=$ $\left\{f_{1 k} \chi_{Q^{*}}, \ldots, f_{m k} \chi_{Q^{*}}\right\}_{k=1}^{\infty}$ and $Q^{*}=(8 \sqrt{n}+4) Q$, then we can write

$$
\begin{aligned}
\prod_{j=1}^{m} f_{j k}\left(y_{j}\right) & =\prod_{j=1}^{m} f_{j k}\left(y_{j}\right)\left(f_{j k}^{0}\left(y_{j}\right)+f_{j k}^{\infty}\left(y_{j}\right)\right) \\
& =\sum_{\alpha_{1}, \ldots, \alpha_{m}} f_{1 k}^{\alpha_{1}}\left(y_{1}\right) \cdots f_{m k}^{\alpha_{m}}\left(y_{m}\right) \\
& =\prod_{j=1}^{m} f_{j k}^{0}\left(y_{j}\right)+\sum^{\prime} f_{1 k}^{\alpha_{1}}\left(y_{1}\right) \cdots f_{m k}^{\alpha_{m}}\left(y_{m}\right),
\end{aligned}
$$

where each term of $\sum^{\prime}$ contains at least one $\alpha_{j} \neq 0$. 
Hence, we can write

$$
T_{q}(\vec{f})(z)=T_{q}\left(\vec{f}^{0}\right)(z)+\sum^{\prime} T_{q}\left(f_{1}^{\alpha_{1}}, \ldots, f_{m}^{\alpha_{m}}\right)(z) .
$$

Applying Kolmogorov's inequality to the term

$$
T_{q}\left(\vec{f}^{0}\right)(z)=T_{q}\left(f_{1 k}^{0}, \ldots, f_{m k}^{0}\right)(z)
$$

with $p=\delta$ and $q=1 / m$, we derive

$$
\begin{aligned}
\left(\frac{1}{|Q|} \int_{Q}\left|T_{q}\left(\vec{f}^{0}\right)(z)\right|^{\delta} d z\right)^{1 / \delta} & \leq C\left\|T_{q}\left(\vec{f}^{0}\right)\right\|_{L^{1 / m, \infty}(\mathrm{Q}, d x /|\mathrm{Q}|)} \\
& \leq C \prod_{j=1}^{m} \frac{1}{\left|Q^{*}\right|} \int_{Q^{*}}\left|f_{j}\right|_{q_{j}} d y_{j} \\
& \leq C \mathscr{M}\left(|\vec{f}|_{q}\right)(x)
\end{aligned}
$$

since $T: L^{1} \times \cdots \times L^{1} \rightarrow L^{1 / m, \infty}$

To estimate the remaining terms, we now set $C_{Q}=\sum^{\prime} T_{q}$ $\left(f_{1 k}^{\alpha_{1}}, \ldots, f_{m k}^{\alpha_{m}}\right)(x)$ and will show that, for any $z \in Q$,

$$
\begin{aligned}
& \sum^{\prime}\left|T_{q}\left(f_{1 k}^{\alpha_{1}}, \ldots, f_{m k}^{\alpha_{m}}\right)(z)-T_{q}\left(f_{1 k}^{\alpha_{1}}, \ldots, f_{m k}^{\alpha_{m}}\right)(x)\right| \\
& \quad \leq \operatorname{CM}\left(|\vec{f}|_{q}\right)(x) .
\end{aligned}
$$

Consider the case when $\alpha_{1}=\cdots=\alpha_{m}=\infty$ and define

$$
T_{q}\left(\vec{f}^{\infty}\right)=T_{q}\left(f_{1 k}^{\infty}, \ldots, f_{m k}^{\infty}\right)
$$

So

$$
\begin{gathered}
\left|T_{q}\left(f_{1 k}^{\infty}, \ldots, f_{m k}^{\infty}\right)(z)-T_{q}\left(f_{1 k}^{\infty}, \ldots, f_{m k}^{\infty}\right)(x)\right| \\
\leq\left(\sum_{k=1}^{\infty} \mid \int_{\left(\mathbb{R}^{n} \backslash Q^{*}\right)^{m}} K(z, \vec{y}) f_{1 k}\left(y_{1}\right) \ldots\right. \\
\left.\left.f_{m k}\left(y_{m}\right) d \vec{y}\right|^{q}\right)^{1 / q} \\
-\left(\sum_{k=1}^{\infty} \mid \int_{\left(\mathbb{R}^{n} \backslash Q^{*}\right)^{m}} K(x, \vec{y}) f_{1 k}\left(y_{1}\right) \ldots\right. \\
\left.\left.f_{m k}\left(y_{m}\right) d \vec{y}\right|^{q}\right)^{1 / q}
\end{gathered}
$$

$$
\begin{aligned}
& \leq\left[\sum _ { k = 1 } ^ { \infty } \left(\int_{\left(\mathbb{R}^{n} \backslash Q^{*}\right)^{m}}|K(z, \vec{y})-K(x, \vec{y})|\right.\right. \\
& \left.\left.\quad \times\left|f_{1 k}\left(y_{1}\right) \cdots f_{m k}\left(y_{m}\right)\right| d \vec{y}\right)^{q}\right]^{1 / q} \\
& \leq\left[\sum _ { k = 1 } ^ { \infty } \left(\int_{\left(\mathbb{R}^{n} \backslash Q^{*}\right)^{m}}\left|K(z, \vec{y})-K_{t}^{(0)}(z, \vec{y})\right|\right.\right. \\
& +\left|K_{t}^{(0)}(z, \vec{y})-K(x, \vec{y})\right| \\
& \left.\left.\quad \times\left|f_{1 k}\left(y_{1}\right) \cdots f_{m k}\left(y_{m}\right)\right| d \vec{y}\right)^{q}\right]^{1 / q} \\
& \leq\left(\sum_{k=1}^{\infty}\left|\sum_{v=0}^{\infty} \int_{\left(2^{v+1} Q^{*} \backslash 2^{v} Q^{*}\right)^{m}}\right| K(z, \vec{y})-K_{t}^{(0)}(z, \vec{y}) \mid\right. \\
& +\left|K_{t}^{(0)}(z, \vec{y})-K(x, \vec{y})\right| \\
& \left.\times\left.\left|f_{1 k}\left(y_{1}\right) \cdots f_{m k}\left(y_{m}\right)\right| d \vec{y}\right|^{q}\right)^{1 / q}
\end{aligned}
$$$$
:=I \text {. }
$$

Since $x, z \in Q, y_{j} \in \mathbb{R}^{n} \backslash(8 \sqrt{n}+4) Q$, and $t=(2 \sqrt{n} l(Q))^{s}$, then $\left|x-y_{j}\right| \sim\left|z-y_{j}\right|,\left|z-y_{j}\right|>(4 \sqrt{n}+1) l(Q)>2 t^{1 / s}$, $\left|x-y_{j}\right|>2 t^{1 / s}$, and $|x-z|<2 t^{1 / s}$. Hence, $\phi\left(\left|y_{j}-z\right| / t^{1 / s}\right)=0$ for $j=1,2$. By Assumption (H2), we can infer

$$
\left|K(z, \vec{y})-K_{t}^{(0)}(z, \vec{y})\right| \leq \frac{A t^{\epsilon / s}}{\left(\sum_{i=1}^{m}\left|z-y_{j}\right|\right)^{m n+\epsilon}},
$$

$$
\left|K_{t}^{(0)}(z, \vec{y})-K(x, \vec{y})\right| \leq \frac{A t^{\epsilon / s}}{\left(\sum_{i=1}^{m}\left|x-y_{j}\right|\right)^{m n+\epsilon}} .
$$

Thus,

$$
\begin{gathered}
I \leq C\left(\sum _ { k = 1 } ^ { \infty } \left(\sum_{\nu=0}^{\infty} \int_{\left(2^{v+1} Q^{*} \backslash 2^{\nu} Q^{*}\right)^{m}} \frac{A t^{\epsilon / s}}{\left(\sum_{i=1}^{m}\left|z-y_{j}\right|\right)^{m n+\epsilon}}\right.\right. \\
\left.\left.\times \prod_{j=1}^{m}\left|f_{j k}\left(y_{j}\right)\right| d \vec{y}\right)^{q}\right)^{1 / q} \\
\leq C\left(\sum _ { k = 1 } ^ { \infty } \left(\sum_{\nu=0}^{\infty} \frac{\left|Q^{*}\right|^{\epsilon / n}\left(2^{n}\left|Q^{*}\right|^{1 / n}\right)^{m n+\epsilon}}{\left.\left.\times \int_{\left(2^{v+1} Q^{*}\right)^{m}} \prod_{j=1}^{m}\left|f_{j k}\left(y_{j}\right)\right| d \vec{y}\right)^{q}\right)^{1 / q}}\right.\right.
\end{gathered}
$$




$$
\begin{aligned}
& \leq C\left(\sum _ { k = 1 } ^ { \infty } \left(\sum_{\nu=0}^{\infty} \frac{1}{2^{\nu \epsilon}} \frac{1}{\left(2^{\nu+1}\left|Q^{*}\right|^{1 / n}\right)^{m n}}\right.\right. \\
& \left.\left.\quad \times \int_{\left(2^{\nu+1} Q^{*}\right)^{m}} \prod_{j=1}^{m}\left|f_{j k}\left(y_{j}\right)\right| d \vec{y}\right)^{q}\right)^{1 / q} \\
& \leq C \sum_{\nu=1}^{\infty} \frac{1}{2^{\nu \epsilon}} \prod_{j=1}^{m}\left[\sum _ { k = 1 } ^ { \infty } \left(\frac{1}{\left(2^{\nu+1}\left|Q^{*}\right|^{1 / n}\right)^{n}}\right.\right. \\
& \leq C \sum_{\nu=1}^{\infty} \frac{1}{2^{\nu \epsilon}} \prod_{j=1}^{m} \frac{1}{\left(2^{\nu+1}\left|Q^{*}\right|^{1 / n}\right)^{n}} \int_{2^{\nu+1} Q^{*}}\left|f_{j}\right|_{q_{j}} d y_{j} \\
& \leq C \cdot \mathscr{M}\left(|\vec{f}|_{q}\right)(x) .
\end{aligned}
$$

It remains to estimate the terms in (46) with $\alpha_{j_{l}}=\cdots=\alpha_{j_{l}}=$ 0 , for some $\varrho=\left\{j_{1}, \ldots, j_{l}\right\} \subset\{1, \ldots, m\}=\varrho_{0}$ for $1 \leq l<m$ and $\varrho^{\prime}=\varrho_{0} \backslash \varrho$, by Assumption (H2), we have

$$
\begin{aligned}
& \left|T_{q}\left(f_{1}^{\alpha_{1}}, \ldots, f_{m}^{\alpha_{m}}\right)(z)-T_{q}\left(f_{1}^{\alpha_{1}}, \ldots, f_{m}^{\alpha_{m}}\right)(x)\right| \\
& \leq\left[\sum _ { k = 1 } ^ { \infty } \left(\int_{\left(\mathbb{R}^{n}\right)^{m}}|K(z, \vec{y})-K(x, \vec{y})|\right.\right. \\
& \left.\left.\times\left|f_{1}^{\alpha_{1}}\left(y_{1}\right) \cdots f_{m}^{\alpha_{m}}\left(y_{m}\right)\right| d \vec{y}\right)^{q}\right]^{1 / q} \\
& \leq\left[\sum _ { k = 1 } ^ { \infty } \left(\int_{\left(\mathbb{R}^{n}\right)^{m}}\left|K(z, \vec{y})-K_{t}^{(0)}(z, \vec{y})\right|\right.\right. \\
& +\left|K_{t}^{(0)}(z, \vec{y})-K(x, \vec{y})\right| \\
& \left.\left.\times\left|f_{1}^{\alpha_{1}}\left(y_{1}\right) \cdots f_{m}^{\alpha_{m}}\left(y_{m}\right)\right| d \vec{y}\right)^{q}\right]^{1 / q} \\
& \leq C\left\{\sum _ { k = 1 } ^ { \infty } \left[\prod_{j \in \varrho}\left|\int_{Q^{*}}\right| f_{j k}\left(y_{j}\right) \mid d y_{j}\right.\right. \\
& \times\left(\int _ { ( \mathbb { R } ^ { n } | Q ^ { * } ) ^ { m } } \left(\frac{t^{\epsilon / t}}{\left(\sum_{j \in Q^{\prime}}\left|z-y_{j}\right|\right)^{m n+\epsilon}}\right.\right. \\
& \left.+\frac{1}{\left(\sum_{j \in Q^{\prime}}\left|z-y_{j}\right|\right)^{m n}}\right) \\
& \left.\left.\left.\times \prod\left|f_{j k}\left(y_{j}\right)\right| d y_{j}\right)\right]^{q}\right\}^{1 / q}
\end{aligned}
$$$$
\leq C\left\{\sum _ { k = 1 } ^ { \infty } \left[\prod_{j \in \varrho} \int_{Q^{*}}\left|f_{j k}\left(y_{j}\right)\right| d y_{j}\right.\right.
$$$$
\times\left(\sum _ { v = 0 } ^ { \infty } \left(\frac{\left|Q^{*}\right|^{\epsilon / n}}{\left(2^{v}\left|Q^{*}\right|^{1 / n}\right)^{m n+\epsilon}}\right.\right.
$$$$
\left.+\frac{1}{\left(2^{v}\left|Q^{*}\right|^{1 / n}\right)^{m n}}\right)
$$$$
\left.\left.\left.\times \int_{\left(2^{v+1} Q^{*}\right)^{m}} \prod_{j \in Q^{\prime}}\left|f_{j k}\left(y_{j}\right)\right| d y_{j}\right)\right]^{q}\right\}^{1 / q}
$$$$
\leq C \sum_{\nu=0}^{\infty}\left(\frac{\left|Q^{*}\right|^{\epsilon / n}}{\left(2^{v}\left|Q^{*}\right|^{1 / n}\right)^{m n+\epsilon}}+\frac{1}{\left(2^{v}\left|Q^{*}\right|^{1 / n}\right)^{m n}}\right)
$$$$
\times \prod_{j \in \varrho}\left[\sum_{k=1}^{\infty}\left(\int_{Q^{*}}\left|f_{j k}\left(y_{j}\right)\right| d y_{j}\right)^{q_{j}}\right]^{1 / q_{j}}
$$$$
\times \prod_{j \in \varrho^{\prime}}\left[\sum_{k=1}^{\infty}\left(\int_{2^{v+1} Q^{*}}\left|f_{j k}\left(y_{j}\right)\right| d y_{j}\right)^{q_{j}}\right]^{1 / q_{j}}
$$$$
\leq C \sum_{v=0}^{\infty} \frac{1}{2^{\nu \epsilon}} \frac{1}{\left(2^{v}\left|Q^{*}\right|^{1 / n}\right)^{m n}} \prod_{j \in \varrho} \int_{Q^{*}}\left|f_{j}\right|_{q_{j}} d y_{j}
$$$$
\times \prod_{j \in \varrho^{\prime}} \int_{2^{2+1} Q^{*}}\left|f_{j}\right|_{q_{j}} d y_{j}
$$$$
+C \sum_{v=0}^{\infty} \frac{1}{\left(2^{v}\left|Q^{*}\right|^{1 / n}\right)^{m n}} \prod_{j \in \varrho} \int_{Q^{*}}\left|f_{j}\right|_{q_{j}} d y_{j}
$$$$
\times \prod_{j \in \varrho^{\prime}} \int_{2^{v+1} Q^{*}}\left|f_{j}\right|_{q_{j}} d y_{j}
$$$$
\leq C \sum_{v=0}^{\infty} \frac{1}{2^{v \epsilon}} \frac{1}{\left(2^{v+1}\left|Q^{*}\right|^{1 / n}\right)^{m n}} \prod_{j=1}^{m} \int_{2^{\nu+1} Q^{*}}\left|f_{j}\right|_{q_{j}} d y_{j}
$$$$
+C \sum_{\gamma=0}^{\infty} \frac{1}{2^{2 n l}} \prod_{j \in \varrho} \frac{1}{\left|Q^{*}\right|} \int_{Q^{*}}\left|f_{j}\right|_{q_{j}} d y_{j}
$$$$
\times \prod_{j \in \varrho^{\prime}} \frac{1}{\left(\left|2^{v+1} Q^{*}\right|^{1 / n}\right)^{n}} \int_{2^{\nu+1} Q^{*}}\left|f_{j}\right|_{q_{j}} d y_{j}
$$$$
\leq C\left(\mathscr{M}\left(|\vec{f}|_{q}\right)(x)+\mathscr{M}_{\varrho}\left(|\vec{f}|_{q}\right)(x)\right) .
$$

This finishes the proof of Proposition 9.

Now we restore to prove Theorem 1. 
Proof of Theorem 1. By the definition of $\vec{\omega} \in A_{\vec{P}}$ implies that $\nu_{\vec{\omega}} \in A_{\infty}$. Using Proposition 9 and the Fefferman-Stein inequality (26) and observing that $0<\delta<1 / m$, we have

$$
\begin{aligned}
\left\|T_{q}(\vec{f})\right\|_{L^{p}\left(v_{\omega}\right)} & \leq\left\|M_{\delta}\left(T_{q}(\vec{f})\right)\right\|_{L^{p}\left(v_{\omega}\right)} \\
\leq & \left\|M_{\delta}^{\sharp}\left(T_{q}(\vec{f})\right)\right\|_{L^{p}\left(v_{\omega}\right)} \\
\leq & \left\|\mathscr{M}\left(|\vec{f}|_{q}\right)\right\|_{L^{p}\left(v_{\omega}\right)} \\
& +\sum_{\varrho \subset \varrho_{0}}\left\|\mathscr{M}_{\varrho}\left(|\vec{f}|_{q}\right)\right\|_{L^{p}\left(v_{\omega}\right)} .
\end{aligned}
$$

Then following from (52) and Lemma 6, Theorem 1 can be proved.

We are left to check that $\left\|M_{\delta}\left(T_{q}(\vec{f})\right)\right\|_{L^{p}\left(v_{\omega}\right)}$ is finite. The method of proof is similar to that of Theorem 3.19 in [2]. So we omit it here.

Here is a crucial proposition on commutator $T_{\Sigma \vec{b}, q}$ to prove Theorem 2 .

Proposition 10. Let $T_{q}$ be a vector-valued multilinear operator associated with an m-linear operator $T$ in $\operatorname{GCZO}(A, s, \eta, \epsilon)$ whose kernels satisfie the Assumption (H2). Suppose that $T_{\Sigma \vec{b}, q}$ is the corresponding commutator of $T_{q}$ with $\vec{b} \in B M O^{m}$. Let $0<\delta<\min \{\epsilon, 1 / m\}, r>1$. Then there exists a constant $C>0$ depending on $\delta$ and $\epsilon$, such that

$$
\begin{aligned}
& M_{\delta}^{\sharp}\left(T_{\Sigma \vec{b}, q}(\vec{f})\right)(x) \\
& \leq C \sum_{i=1}^{m}\left(M_{\epsilon}\left(T_{q}(\vec{f})\right)(x)+M_{L(\log L)}\left(|\vec{f}|_{q}\right)(x)\right. \\
&\left.\quad+\sum_{\emptyset \neq \varrho \subset \varrho_{0}} \mathscr{M}_{\varrho, L(\log L)}\left(|\vec{f}|_{q}\right)(x)\right)
\end{aligned}
$$

holds for all bounded measurable vector functions $\left\{\vec{f}_{k}\right\}_{k=1}^{\infty}=$ $\left\{f_{1 k}, \ldots, f_{m k}\right\}_{k=1}^{\infty}$.

Proof of Proposition 10. By linearity, it suffices to consider the operator with only symbols. Without loss of generality, we only consider the case: $j=1$ and denote $b_{1}$ by $b$ for convenience.

Note that for any constant $\lambda$ we have

$$
\begin{aligned}
T_{\Sigma \vec{b}, q}(\vec{f})(z)= & (b(z)-\lambda) T_{q}\left(f_{1}, \ldots, f_{m}\right)(z) \\
& -T_{q}\left(b f_{1}, \ldots, f_{m}\right)(z) .
\end{aligned}
$$

Fix $x$, for any cube $Q$ centered at $x$ and a constant $c$ determined later, we have

$$
\begin{aligned}
& \left(\frac{1}{|Q|} \int_{Q}\left|T_{b, q}(\vec{f})(z)\right|^{\delta}-|c|^{\delta} d z\right)^{1 / \delta} \\
& \quad \leq\left(\frac{1}{|Q|} \int_{Q}\left|T_{b, q}(\vec{f})(z)-c\right|^{\delta} d z\right)^{1 / \delta}
\end{aligned}
$$

$$
\begin{aligned}
\leq & \left(\frac{C}{|Q|} \int_{Q}\left|(b(z)-\lambda) T_{q}(\vec{f})(z)\right|^{\delta} d z\right)^{1 / \delta} \\
& +\left(\frac{C}{|Q|} \int_{Q}\left|T_{q}\left((b-\lambda) f_{1}, \ldots, f_{m}\right)(z)-c\right|^{\delta} d z\right)^{1 / \delta} \\
:= & I+I I .
\end{aligned}
$$

We analyze each term separately. Recall that $Q^{*}=(8 \sqrt{n}+4) Q$ and $\lambda=b_{\mathrm{Q}^{*}}$ and thanks to Hölder's inequality and note that $1<q<\epsilon / \delta$, it follows that

$$
\begin{aligned}
I \leq & C\left(\frac{1}{|Q|} \int_{Q}|b(z)-\lambda|^{\delta q^{\prime}} d z\right)^{1 / \delta q^{\prime}} \\
& \times\left(\frac{1}{|Q|} \int_{Q}\left|T_{q}(\vec{f})(z)\right|^{\delta q} d z\right)^{1 / \delta q} \\
\leq & C\|b\|_{\mathrm{BMO}} M_{\epsilon}\left(T_{q}(\vec{f})\right)(x) .
\end{aligned}
$$

To estimate $I I$, we split again each $\vec{f}_{k}$ into $\vec{f}_{k}^{0}+\vec{f}_{k}^{\infty}$, where $\vec{f}_{k}^{0}=\vec{f}_{k} \chi_{\mathrm{Q}^{*}}=\left\{f_{1 k} \chi_{\mathrm{Q}^{*}}, \ldots, f_{m k} \chi_{\mathrm{Q}^{*}}\right\}$. This yields

$$
\begin{aligned}
\prod_{j=1}^{m} f_{j k}\left(y_{j}\right) & =\sum_{\left\{\alpha_{1}, \ldots, \alpha_{m}\right\} \in\{0, \infty\}} f_{\alpha_{1}}\left(y_{1}\right) \cdots f_{m k}^{\alpha_{m}}\left(y_{m}\right) \\
& =\prod_{j=1}^{m} f_{j k}^{0}\left(y_{j}\right)+\sum^{\prime} f_{1 k}^{\alpha_{1}}\left(y_{1}\right) \cdots f_{m k}^{\alpha_{m}}\left(y_{m}\right),
\end{aligned}
$$

where each term in $\sum^{\prime}$ contains at least one $\alpha_{j} \neq 0$.

Choose $c=\sum^{\prime} C_{\alpha_{1}, \ldots, \alpha_{m}}$ with $C_{\alpha_{1}, \ldots, \alpha_{m}}=T_{q}\left((b-\lambda) f_{1 k}^{\alpha_{1}}, \ldots\right.$, $\left.f_{m k}^{\alpha_{m}}\right)(x)$, then

$$
\begin{aligned}
I I \leq & C\left(\frac{1}{|Q|} \int_{Q}\left|T_{q}\left((b-\lambda) f_{1 k}^{0}, \ldots, f_{m k}^{0}\right)(z)\right|^{\delta} d z\right)^{1 / \delta} \\
& +\sum^{\prime}\left(\frac{1}{|Q|} \int_{Q} \mid T_{q}\left((b-\lambda) f_{1 k}^{\alpha_{1}}, \ldots, f_{m k}^{\alpha_{m}}\right)(z)\right. \\
& \left.-\left.C_{\alpha_{1}, \ldots, \alpha_{m}}\right|^{\delta} d z\right)^{1 / \delta} \\
:= & I I_{0}+\sum^{\prime} I I_{\alpha_{1}, \ldots, \alpha_{m}} .
\end{aligned}
$$

Noting that $\delta<1 / m$ and $T_{q}: L^{1} \times \cdots \times L^{1} \rightarrow L^{1 / m, \infty}$ and using Hölder' inequality, we obtain

$$
\begin{aligned}
I I_{0} & =C\left(\frac{1}{|Q|} \int_{Q}\left|T_{q}\left((b-\lambda) f_{1 k}^{0}, \ldots, f_{m k}^{0}\right)(z)\right|^{\delta} d z\right)^{1 / \delta} \\
& \leq C\left\|T_{q}\left((b-\lambda) f_{1 k}^{0}, \ldots, f_{m k}^{0}\right)\right\|_{L^{1 / m, \infty}(Q, d x /|Q|)}
\end{aligned}
$$




$$
\begin{aligned}
& \leq C \frac{1}{|Q|} \int_{Q}\left|b\left(y_{1}\right)-\lambda\right|\left|f_{1}^{0}\right|_{q} d y_{1} \prod_{j=2}^{m} \frac{1}{|Q|} \int_{Q}\left|f_{j}^{0}\right|_{q_{j}} d y_{j} \\
& \leq C \frac{1}{|Q|} \int_{Q^{*}}\left|b\left(y_{1}\right)-\lambda\right|\left|f_{1}\right|_{q} d y_{1} \prod_{j=2}^{m} \frac{1}{|Q|} \int_{Q^{*}}\left|f_{j}\right|_{q_{j}} d y_{j} \\
& \leq C\|b\|_{\mathrm{BMO}}\left\|\left|f_{1}\right|_{q}\right\|_{L(\log L), \mathrm{Q}^{*}} \prod_{j=2}^{m}\left(\left|f_{j}\right|_{q_{j}}\right)_{\mathrm{Q}^{*}} \\
& \leq C\|b\|_{\mathrm{BMO}} \mathscr{M}_{L(\log L)}\left(|\vec{f}|_{q}\right)(x) .
\end{aligned}
$$

Now consider the term $I I_{\infty, \ldots, \infty}$. By Hölder's and Minkoswki's inequalities as well as Assumptions (H1) and (H2), we obtain

$$
\begin{aligned}
& I I_{\infty, \ldots, \infty} \\
& =\left(\frac{1}{|Q|} \int_{Q} \mid T_{q}\left((b-\lambda) f_{1 k}^{\infty}, \ldots, f_{m k}^{\infty}\right)(z)\right. \\
& \left.-\left.T_{q}\left((b-\lambda) f_{1 k}^{\infty}, \ldots, f_{m k}^{\infty}\right)(x)\right|^{\delta} d z\right)^{1 / \delta} \\
& \leq C \frac{1}{|Q|} \int_{Q}\left[\sum _ { k = 1 } ^ { \infty } \left(\int_{\left(\mathbb{R}^{n} \mid Q^{*}\right)^{m}}|K(z, \vec{y})-K(x, \vec{y})|\right.\right. \\
& \times\left|b\left(y_{1}\right)-\lambda\right|\left|f_{1 k}\left(y_{1}\right)\right| \\
& \left.\left.\times \prod_{j=2}^{m}\left|f_{j k}\left(y_{j}\right)\right| d \vec{y}\right)^{q}\right]^{1 / q} d z \\
& \leq C \frac{1}{|Q|} \int_{Q}\left[\sum _ { k = 1 } ^ { \infty } \left(\int _ { ( \mathbb { R } ^ { n } \backslash Q ^ { * } ) ^ { m } } \left(\left|K(z, \vec{y})-K_{t}^{(0)}(z, \vec{y})\right|\right.\right.\right. \\
& \left.+\left|K_{t}^{(0)}(z, \vec{y})-K(x, \vec{y})\right|\right) \\
& \times\left|b\left(y_{1}\right)-\lambda\right|\left|f_{1 k}\left(y_{1}\right)\right| \\
& \left.\left.\times \prod_{j=2}^{m}\left|f_{j k}\left(y_{j}\right)\right| d \vec{y}\right)^{q}\right]^{1 / q} d z
\end{aligned}
$$$$
:=I I_{\infty, \ldots, \infty}^{\prime} .
$$

Since $x, z \in Q, y_{j} \in \mathbb{R}^{n} \backslash(8 \sqrt{n}+4) Q$, and $t=(2 \sqrt{n} l(Q))^{s}$, then $\left|x-y_{j}\right| \sim\left|z-y_{j}\right|,\left|z-y_{j}\right|>(4 \sqrt{n}+1) l(Q)>2 t^{1 / s}$, $\left|x-y_{j}\right|>2 t^{1 / s}$ and $|x-z|<2 t^{1 / s}$. Hence, $\phi\left(\left|y_{j}-z\right| / t^{1 / s}\right)=0$ for $j=1,2$.

Thus from the Assumption (H2), we can follow that

$$
\begin{array}{r}
I I_{\infty, \ldots, \infty}^{\prime} \leq \frac{C}{|Q|} \int_{Q}\left[\sum _ { k = 1 } ^ { \infty } \left(\int_{\left(\mathbb{R}^{n} \backslash Q^{*}\right)^{m}} \frac{A t^{\epsilon / s}}{\left(\sum_{i=1}^{m}\left|z-y_{j}\right|\right)^{m n+\epsilon}}\right.\right. \\
\times\left|b\left(y_{1}\right)-\lambda\right|\left|f_{1 k}\left(y_{1}\right)\right|
\end{array}
$$

$$
\begin{aligned}
\leq C\left[\sum_{k=1}^{\infty}\left(\sum_{\nu=0}^{\infty} \frac{\left|Q^{*}\right|^{\epsilon=2}}{\left(2^{v}\left|Q^{*}\right|^{1 / n}\right)^{m n+\epsilon}}\left|f_{j k}\left(y_{j}\right)\right| d \vec{y}\right)^{q}\right]^{1 / q} d z \\
\times \int_{\left(2^{v+1} Q^{*}\right)^{m}}\left|b_{1}\left(y_{1}\right)-\lambda\right|\left|f_{1}\left(y_{1}\right)\right| \\
\left.\left.\times \prod_{j=1}^{m}\left|f_{j}\left(y_{j}\right)\right| d \vec{y}\right)^{q}\right]^{1 / q}
\end{aligned}
$$$$
\leq C \sum_{\nu=0}^{\infty} \frac{1}{2^{\nu \epsilon}} \frac{1}{\left(2^{\nu+1}\left|Q^{*}\right|^{1 / n}\right)^{m n}}
$$$$
\times\left[\sum _ { k = 1 } ^ { \infty } \left(\int_{2^{v+1} Q^{*}}\left|b_{1}\left(y_{1}\right)-\lambda\right|\right.\right.
$$$$
\left.\left.\times\left|f_{1}\left(y_{1}\right)\right| d y_{1}\right)^{q_{1}}\right]^{1 / q_{1}}
$$$$
\times \prod_{j=2}^{m}\left[\sum_{k=1}^{\infty}\left(\int_{2^{v+1} Q^{*}}\left|f_{j}\left(y_{j}\right)\right| d y_{j}\right)^{q_{j}}\right]^{1 / q_{j}}
$$$$
\leq C \sum_{v=0}^{\infty} \frac{1}{2^{v \epsilon}} \frac{1}{\left(2^{v+1}\left|Q^{*}\right|^{1 / n}\right)^{m n}}
$$$$
\times \int_{2^{v+1} Q^{*}}\left|b_{1}\left(y_{1}\right)-\lambda\right|\left|f_{1}\right|_{q_{1}} d y_{1}
$$$$
\times \prod_{j=2}^{m} \int_{2^{v+1} Q^{*}}\left|f_{j}\right|_{q_{j}} d y_{j}
$$$$
\leq C \sum_{v=0}^{\infty} \frac{v}{2^{\nu \epsilon}}\|b\|_{\mathrm{BMO}}\left\|\left|f_{1}\right|_{q_{1}}\right\|_{L(\log L), 2^{v+1} \mathrm{Q}^{*}}
$$$$
\times \prod_{j=2}^{m}\left\|\left|f_{j}\right|_{q_{j}}\right\|_{L(\log L), 2^{\nu+1} Q^{*}}
$$$$
\leq C\|b\|_{\mathrm{BMO}} \mathscr{M}_{L(\log L)}\left(|\vec{f}|_{q}\right)(x),
$$

where we use the fact that $\|b-\lambda\|_{\exp L, 2^{\nu+1} \mathrm{Q}} \leq C \nu\|b\|_{\exp L, Q}$.

What remains to be considered is the term $I I_{\alpha_{1}, \ldots, \alpha_{m}}$ such that $\alpha_{j_{1}}=\cdots=\alpha_{j_{l}}=0$, for some $\varrho=\left\{j_{1}, \ldots, j_{l}\right\} \subset\{1, \ldots, m\}$ for $1 \leq l<m$ and denote $\varrho^{\prime}=\varrho_{0} \backslash \varrho$. We consider only the case $1 \in \varrho$, by Assumption (H2), we have

$$
\begin{aligned}
I I_{\alpha_{1}, \ldots, \alpha_{m}}=\left(\frac{1}{|Q|} \int_{Q} \mid\right. & T_{q}\left((b-\lambda) f_{1 k}^{\alpha_{1}}, \ldots, f_{m k}^{\alpha_{m}}\right)(z) \\
& \left.\quad-\left.T_{q}\left((b-\lambda) f_{1 k}^{\alpha_{1}}, \ldots, f_{m k}^{\alpha_{m}}\right)(x)\right|^{\delta} d z\right)^{1 / \delta}
\end{aligned}
$$




$$
\begin{aligned}
& \leq \frac{1}{|Q|} \int_{Q} \mid T_{q}\left((b-\lambda) f_{1 k}^{\alpha_{1}}, \ldots, f_{m k}^{\alpha_{m}}\right)(z) \\
& -T_{q}\left((b-\lambda) f_{1 k}^{\alpha_{1}}, \ldots, f_{m k}^{\alpha_{m}}\right)(x) \mid d z \\
& \leq \frac{1}{|Q|} \int_{Q}\left[\sum _ { k = 1 } ^ { \infty } \left(\int_{\left(\mathbb{R}^{n}\right)^{m}}|K(z, \vec{y})-K(x, \vec{y})|\right.\right. \\
& \times\left|b\left(y_{1}\right)-\lambda\right|\left|f_{1 k}^{0}\left(y_{1}\right)\right| \\
& \left.\left.\times \prod_{j=2}^{m}\left|f_{j k}^{\alpha_{j}}\left(y_{j}\right)\right| d \vec{y}\right)^{q}\right]^{1 / q} d z \\
& \leq \frac{1}{|Q|} \int_{Q}\left[\sum _ { k = 1 } ^ { \infty } \left(\int _ { ( \mathbb { R } ^ { n } ) ^ { m } } \left(\left|K(z, \vec{y})-K_{t}^{(0)}(x, \vec{y})\right|\right.\right.\right. \\
& \left.+\left|K_{t}^{(0)}(z, \vec{y})-K(x, \vec{y})\right|\right) \\
& \times\left|b\left(y_{1}\right)-\lambda\right|\left|f_{1 k}^{0}\left(y_{1}\right)\right| \\
& \left.\left.\times \prod_{j=2}^{m}\left|f_{j k}^{\alpha_{j}}\left(y_{j}\right)\right| d \vec{y}\right)^{q}\right]^{1 / q} d z \\
& \leq \frac{C}{|Q|} \int_{Q}\left[\sum _ { k = 1 } ^ { \infty } \left(\int_{Q^{*}}\left|b\left(y_{1}\right)-\lambda\right|\left|f_{1 k}\left(y_{1}\right)\right| d y_{1}\right.\right. \\
& \times \prod_{j \in \varrho \backslash\{1\}} \int_{Q^{*}}\left|f_{j k}\left(y_{j}\right)\right| d y_{j} \\
& \times \int_{\left(\mathbb{R}^{n} \backslash Q^{*}\right)^{m-l}} \\
& \times\left(\frac{t^{\epsilon / t}}{\left(\sum_{j \in Q^{\prime}}\left|z-y_{j}\right|\right)^{m n+\epsilon}}\right. \\
& \left.+\frac{1}{\left(\sum_{j \in \varrho^{\prime}}\left|z-y_{j}\right|\right)^{m n}}\right) \\
& \left.\left.\times \prod_{j \in \varrho^{\prime}}\left|f_{j k}\left(y_{j}\right)\right| d y_{j}\right)^{q}\right]^{1 / q} d z \\
& \leq \frac{C}{|Q|} \int_{Q}\left[\sum _ { k = 1 } ^ { \infty } \left(\int_{Q^{*}}\left|b\left(y_{1}\right)-\lambda\right|\left|f_{1 k}\left(y_{1}\right)\right| d y_{1}\right.\right. \\
& \times \prod_{j \in \varrho \backslash\{1\}} \int_{Q^{*}}\left|f_{j k}\left(y_{j}\right)\right| d y_{j} \\
& \times \sum_{\nu=0}^{\infty} \int_{\left(2^{v+1} Q^{*} \mid 2^{v} Q^{*}\right)^{m-l}} \\
& \times\left(\frac{t^{\epsilon / t}}{\left(\sum_{j \in Q^{\prime}}\left|z-y_{j}\right|\right)^{m n+\epsilon}}\right. \\
& \left.+\frac{1}{\left(\sum_{j \in \varrho^{\prime}}\left|z-y_{j}\right|\right)^{m n}}\right) \\
& \left.\left.\times \prod_{j \in \varrho^{\prime}}\left|f_{j k}\left(y_{j}\right)\right| d y_{j}\right)^{q}\right]^{1 / q} d z \\
& \leq C\left[\sum _ { k = 1 } ^ { \infty } \left(\int_{Q^{*}}\left|b\left(y_{1}\right)-\lambda\right|\left|f_{1 k}\left(y_{1}\right)\right| d y_{1}\right.\right. \\
& \times \prod_{j \in Q \backslash\{1\}} \int_{\mathrm{Q}^{*}}\left|f_{j k}\left(y_{j}\right)\right| d y_{j} \\
& \times \sum_{\gamma=0}^{\infty}\left(\frac{\left|Q^{*}\right|^{\epsilon / n}}{\left(2^{v}\left|Q^{*}\right|^{1 / n}\right)^{m n+\epsilon}}\right. \\
& \left.+\frac{1}{\left(2^{v}\left|Q^{*}\right|^{1 / n}\right)^{m n}}\right) \\
& \left.\left.\times \int_{\left(2^{p+1} Q^{*}\right)^{m-l}} \prod_{j \in \varrho^{\prime}}\left|f_{j k}\left(y_{j}\right)\right| d y_{j}\right)^{q}\right]^{1 / q} \\
& \leq C\left[\sum_{k=1}^{\infty}\left(\int_{Q^{*}}\left|b\left(y_{1}\right)-\lambda\right|\left|f_{1 k}\left(y_{1}\right)\right| d y_{1}\right)^{q_{1}}\right]^{1 / q_{1}} \\
& \times \prod_{j \in \varrho \backslash\{1\}}\left[\sum_{k=1}^{\infty}\left(\int_{Q^{*}}\left|f_{j k}\left(y_{j}\right)\right| d y_{j}\right)^{q_{j}}\right]^{1 / q_{j}} \\
& \times \sum_{\nu=0}^{\infty}\left(\frac{\left|Q^{*}\right|^{\epsilon / n}}{\left(2^{\nu}\left|Q^{*}\right|^{1 / n}\right)^{m n+\epsilon}}+\frac{1}{\left(2^{\nu}\left|Q^{*}\right|^{1 / n}\right)^{m n}}\right) \\
& \times \prod_{j \in \varrho^{\prime}}\left[\sum_{k=1}^{\infty}\left(\int_{2^{v+1} Q^{*}}\left|f_{j k}\left(y_{j}\right)\right| d y_{j}\right)^{q_{j}}\right]^{1 / q_{j}} \\
& \leq \int_{Q^{*}}\left|b\left(y_{1}\right)-\lambda\right|\left|f_{1}\right|_{q_{1}} d y_{1} \\
& \times \prod_{j \in \varrho \backslash\{1\}} \int_{Q^{*}}\left|f_{j}\right|_{q_{j}} d y_{j} \\
& \times \sum_{\nu=0}^{\infty}\left(\frac{1}{2^{\nu \epsilon}} \frac{1}{\left(2^{v}\left|Q^{*}\right|^{1 / n}\right)^{m n}}\right. \\
& \left.+\frac{1}{\left(2^{v}\left|Q^{*}\right|^{1 / n}\right)^{m n}}\right) \\
& \times \prod_{j \in \varrho^{\prime}} \int_{2^{v+1} Q^{*}}\left|f_{j}\right|_{q_{j}} d y_{j} \\
& \leq C\|b\|_{\mathrm{BMO}}\left\|\left|f_{1}\right|_{q_{1}}\right\|_{L(\log L), \mathrm{Q}^{*}}\left|Q^{*}\right| \\
& \times \prod_{j \in \varrho \backslash\{1\}} \int_{Q^{*}}\left|f_{j}\right|_{q_{j}} d y_{j}
\end{aligned}
$$




$$
\begin{gathered}
\times\left[\sum_{v=0}^{\infty} \frac{1}{2^{v \epsilon}} \frac{1}{\left(2^{v}\left|Q^{*}\right|^{1 / n}\right)^{m n}}\right. \\
\times \prod_{j \in \varrho^{\prime}} \int_{2^{v+1} Q^{*}}\left|f_{j}\right|_{q_{j}} d y_{j} \\
+\sum_{v=0}^{\infty} \frac{1}{\left(2^{v}\left|Q^{*}\right|^{1 / n}\right)^{m n}} \\
\left.\times \prod_{j \in \varrho^{\prime}} \int_{2^{v+1} Q^{*}}\left|f_{j}\right|_{q_{j}} d y_{j}\right] \\
\leq C\|b\|_{\mathrm{BMO}}\left(\sum_{v=0}^{\infty} \frac{1}{2^{v \epsilon}} \prod_{j=1}^{m} \|\left.\left|f_{j}\right|_{q_{j}}\right|_{L(\log L), 2^{v+1} Q^{*}}\right. \\
+\sum_{v=0}^{\infty} \frac{1}{2^{v n l}} \prod_{j \in \varrho} \|\left.\left|f_{j}\right|_{q_{j}}\right|_{L(\log L), Q^{*}} \\
\left.\times\left.\prod_{j \in \varrho^{\prime}}|| f_{j}\right|_{q_{j}} \|_{L(\log L), 2^{v+1} Q^{*}}\right) \\
\leq C\|b\|_{\mathrm{BMO}}\left(\mathscr{M}_{L(\log L)}\left(|\vec{f}|_{q}\right)(x)\right. \\
\left.+\mathscr{M}_{Q, L(\log L)}\left(|\vec{f}|_{q}\right)(x)\right) .
\end{gathered}
$$

This finishes the proof of Proposition 10.

The next paragraph we will present the proof of Theorem 2.

Proof of Theorem 2. By Lemma 4, $\vec{\omega} \in A_{\vec{p}}$ implies that $\nu_{\vec{\omega}} \in$ $A_{\infty}$. For simplicity, we may assume that $\sum_{i=1}^{m}\left\|b_{i}\right\|_{\mathrm{BMO}}=1$. Using Proposition 10 and the Fefferman-Stein inequality (26), with $0<\delta<\epsilon<1 / m$, we have

$$
\begin{aligned}
& \left\|T_{\Sigma \vec{b}, q}(\vec{f})\right\|_{L^{p}\left(v_{\omega}\right)} \\
& \leq C\left\|M_{\delta}\left(T_{\Sigma \vec{b}, q}(\vec{f})\right)\right\|_{L^{p}\left(v_{\omega}\right)} \\
& \leq C\left\|M_{\delta}^{\sharp}\left(T_{\Sigma \vec{b}, q}(\vec{f})\right)\right\|_{L^{p}\left(v_{\omega}\right)} \\
& \leq C\left(\left\|M_{\epsilon}\left(T_{q}(\vec{f})\right)\right\|_{L^{p}\left(v_{\omega}\right)}+\left\|M_{L(\log L)}\left(|\vec{f}|_{q}\right)\right\|_{L^{p}\left(v_{\omega}\right)}\right. \\
& \left.\quad+\sum_{\varrho \subset \varrho_{0}}\left\|M_{\varrho, L(\log L)}\left(|\vec{f}|_{q}\right)\right\|_{L^{p}\left(v_{\omega}\right)}\right) \\
& \quad C \quad\left\|M_{\epsilon}^{\sharp}\left(T_{q}(\vec{f})\right)\right\|_{L^{p}\left(v_{\omega}\right)}+\left\|\mathscr{M}_{L(\log L)}\left(|\vec{f}|_{q}\right)\right\|_{L^{p}\left(v_{\omega}\right)} \\
& \left.\quad+\sum_{\varrho \subset \varrho_{0}}\left\|\mathscr{M}_{\varrho, L(\log L)}\left(|\vec{f}|_{q}\right)\right\|_{L^{p}\left(v_{\omega}\right)}\right)
\end{aligned}
$$

$$
\begin{gathered}
\leq C\left(\left\|\mathscr{M}\left(|\vec{f}|_{q}\right)\right\|_{L^{p}\left(v_{\omega}\right)}+\left\|\mathscr{M}_{Q}\left(|\vec{f}|_{q}\right)\right\|_{L^{p}\left(v_{\omega}\right)}\right. \\
+\left\|\mathscr{M}_{L(\log L)}\left(|\vec{f}|_{q}\right)\right\|_{L^{p}\left(v_{\omega}\right)} \\
\left.+\sum_{\varrho \subset \varrho_{0}}\left\|\mathscr{M}_{\varrho, L(\log L)}\left(|\vec{f}|_{q}\right)\right\|_{L^{p}\left(v_{\omega}\right)}\right)
\end{gathered}
$$

Then following from (63) and Lemma 5, Theorem 2 is proved.

The reminder that we are left to do is similar to the proof of Theorem 1 . Here we omit it.

The proof of Theorem 3 heavily relied on Proposition 11.

Proposition 11. Let $T_{q}$ be a vector-valued multilinear operator associated with an m-linear operator $T$ in $\operatorname{GCZO}(A, s, \eta, \epsilon)$ whose kernel satisfies the Assumption (H2). Suppose that $T_{\Pi \vec{b}, q}$ is the corresponding iterated commutator of $T_{q}$ with $\vec{b} \in$ $B M O^{2}$. Let $0<\delta<\min \{\epsilon, 1 / 6\}, r>1$, and $\varrho \subset \varrho_{0}=$ $\{1, \ldots, m\}$. Then there exists a constant $C>0$ depending on $\delta$ and $\epsilon$, such that

$$
\begin{aligned}
M_{\delta}^{\sharp}\left(T_{\Pi \vec{b}}(\vec{f})\right)(x) & \\
\leq & C\left\|b_{1}\right\|_{B M O}\left\|b_{2}\right\|_{B M O} \\
& \times\left(M_{\epsilon}\left(T_{q}(\vec{f})\right)(x)+\mathscr{M}_{L(\log L)}\left(|\vec{f}|_{q}\right)(x)\right. \\
& \left.\quad+\mathscr{M}_{\{1\}, L(\log L)}\left(|\vec{f}|_{q}\right)(x)+\mathscr{M}_{\{2\}, L(\log L)}\left(|\vec{f}|_{q}\right)(x)\right) .
\end{aligned}
$$

The proof of Proposition 11 may be omitted since it is easy to get by the similar arguments of the proof of Proposition 10 and [3].

Finally we will give the proof of Theorem 3 .

Proof of Theorem 3. By Lemma 4, $\vec{\omega} \in A_{\vec{p}}$ implies that $\nu_{\vec{\omega}} \in$ $A_{\infty}$. For simplicity, we may assume that $\sum_{i=1}^{m}\left\|b_{i}\right\|_{\mathrm{BMO}}=1$. Using Proposition 11 and the Fefferman-Stein inequality (26) with $0<\delta<\epsilon<1 / m$, we have

$$
\begin{aligned}
& \left\|T_{\Pi \vec{b}, q}(\vec{f})\right\|_{L^{p}\left(v_{\omega}\right)} \\
& \leq C\left\|M_{\delta}\left(T_{\Pi \vec{b}, q}(\vec{f})\right)\right\|_{L^{p}\left(v_{\omega}\right)} \\
& \leq C\left\|M_{\delta}^{\sharp}\left(T_{\Pi \vec{b}, q}(\vec{f})\right)\right\|_{L^{p}\left(v_{\omega}\right)} \\
& \leq C\left(\left\|M_{\epsilon}\left(T_{q}(\vec{f})\right)\right\|_{L^{p}\left(v_{\omega}\right)}+\left\|M_{L(\log L)}\left(|\vec{f}|_{q}\right)\right\|_{L^{p}\left(v_{\omega}\right)}\right. \\
& \left.\quad+\sum_{\varrho \subset \varrho_{0}}\left\|M_{Q, L(\log L)}\left(|\vec{f}|_{q}\right)\right\|_{L^{p}\left(v_{\omega}\right)}\right)
\end{aligned}
$$




$$
\begin{gathered}
\leq C\left(\left\|M_{\epsilon}^{\sharp}\left(T_{q}(\vec{f})\right)\right\|_{L^{p}\left(v_{\omega}\right)}+\left\|\mathscr{M}_{L(\log L)}\left(|\vec{f}|_{q}\right)\right\|_{L^{p}\left(v_{\omega}\right)}\right. \\
\left.\quad+\sum_{\varrho \subset \varrho_{0}}\left\|\mathscr{M}_{Q, L(\log L)}\left(|\vec{f}|_{q}\right)\right\|_{L^{p}\left(v_{\omega}\right)}\right) \\
\leq C\left(\left\|\mathscr{M}\left(|\vec{f}|_{q}\right)\right\|_{L^{p}\left(v_{\omega}\right)}+\left\|\mathscr{M}_{\varrho}\left(|\vec{f}|_{q}\right)\right\|_{L^{p}\left(v_{\omega}\right)}\right. \\
+\left\|\mathscr{M}_{L(\log L)}\left(|\vec{f}|_{q}\right)\right\|_{L^{p}\left(v_{\omega}\right)} \\
\left.+\sum_{\varrho \subset \varrho_{0}}\left\|\mathscr{M}_{\varrho, L(\log L)}\left(|\vec{f}|_{q}\right)\right\|_{L^{p}\left(v_{\omega}\right)}\right) .
\end{gathered}
$$

Then following from (65) and Lemma 5, Theorem 3 is proved.

How to check that $\left\|M_{\delta}\left(T_{\Sigma \vec{b}, q}(\vec{f})\right)\right\|_{L^{p}\left(\nu_{\omega}\right)}$ and $\left\|M_{\epsilon}\left(T_{q}(\vec{f})\right)\right\|_{L^{p}\left(v_{\omega}\right)}$ are finite is similar to the proof of Theorem 3.19 in [2]. We omit it here, too.

\section{References}

[1] A. K. Lerner, S. Ombrosi, C. Pérez, R. H. Torres, and R. TrujilloGonzález, "New maximal functions and multiple weights for the multilinear Calderón-Zygmund theory," Advances in Mathematics, vol. 220, no. 4, pp. 1222-1264, 2009.

[2] S. Chen and H. X. Wu, "Multiple weighted estimates for commutators of multilinear singular integrals with non-smooth kernels," Journal of Mathematical Analysis and Applications, vol. 396, no. 2, pp. 888-903, 2012.

[3] B. T. Anh and X. T. Duong, "On commutators of vector BMO functions and multilinear singular integrals with non-smooth kernels," Journal of Mathematical Analysis and Applications, vol. 371, no. 1, pp. 80-94, 2010.

[4] L. Grafakos and R. H. Torres, "Multilinear Calderón-Zygmund theory," Advances in Mathematics, vol. 165, no. 1, pp. 124-164, 2002.

[5] X. T. Duong, L. Grafakos, and L. X. Yan, "Multilinear operators with non-smooth kernels and commutators of singular integrals," Transactions of the American Mathematical Society, vol. 362, no. 4, pp. 2089-2113, 2010.

[6] L. Tang, "Weighted estimates for vector-valued commutators of multilinear operators," Proceedings of the Royal Society of Edinburgh A, vol. 138, no. 4, pp. 897-922, 2008.

[7] L. Grafakos and J. M. Martell, "Extrapolation of weighted norm inequalities for multivariable operators and applications," The Journal of Geometric Analysis, vol. 14, no. 1, pp. 19-46, 2004.

[8] D. Cruz-Uribe, J. M. Martell, and C. Pérez, "Extrapolation from $A_{\infty}$ weights and applications," Journal of Functional Analysis, vol. 213, no. 2, pp. 412-439, 2004.

[9] C. Fefferman and E. M. Stein, " $H^{p}$ spaces of several variables," Acta Mathematica, vol. 129, no. 3-4, pp. 137-193, 1972.

[10] L. Grafakos, L. G. Liu, and D. C. Yang, "Multiple-weighted norm inequalities for maximal multi-linear singular integrals with non-smooth kernels," Proceedings of the Royal Society of Edinburgh A, vol. 141, no. 4, pp. 755-775, 2011. 


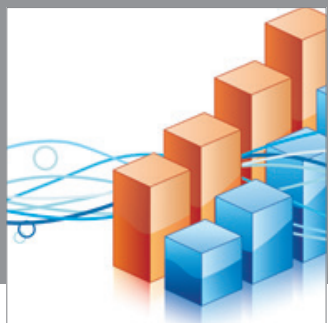

Advances in

Operations Research

mansans

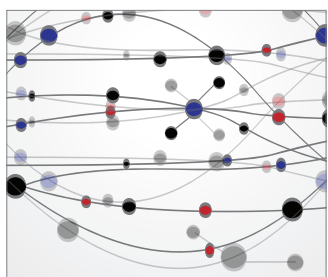

The Scientific World Journal
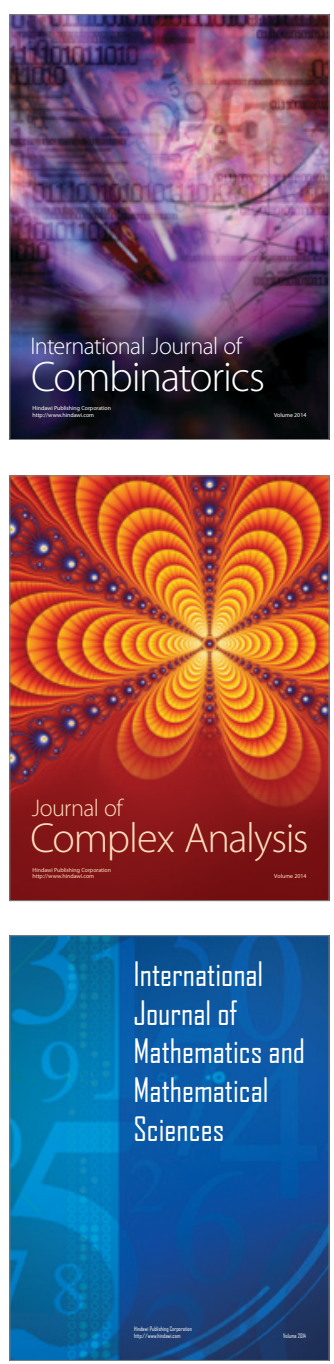
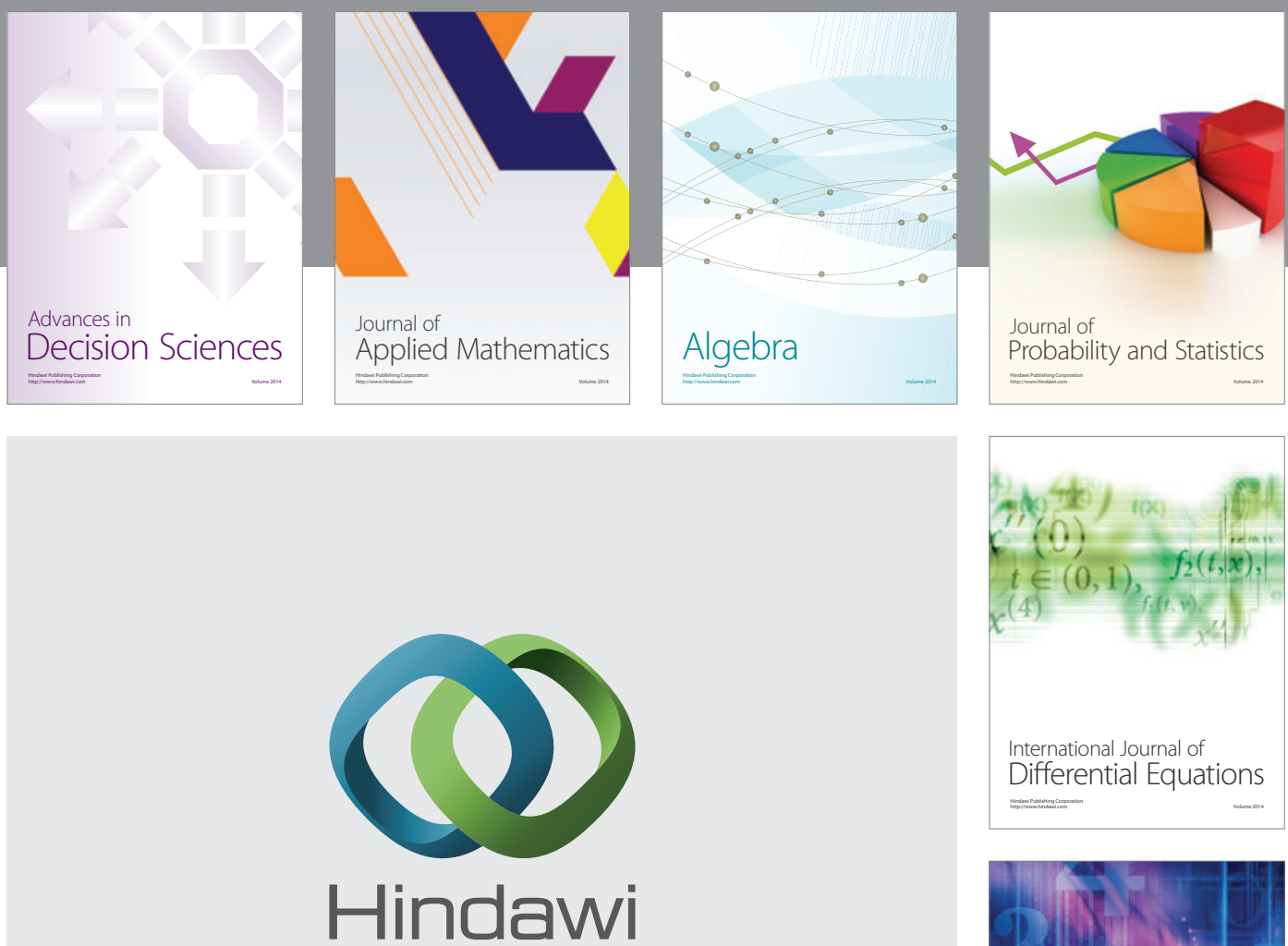

Submit your manuscripts at http://www.hindawi.com
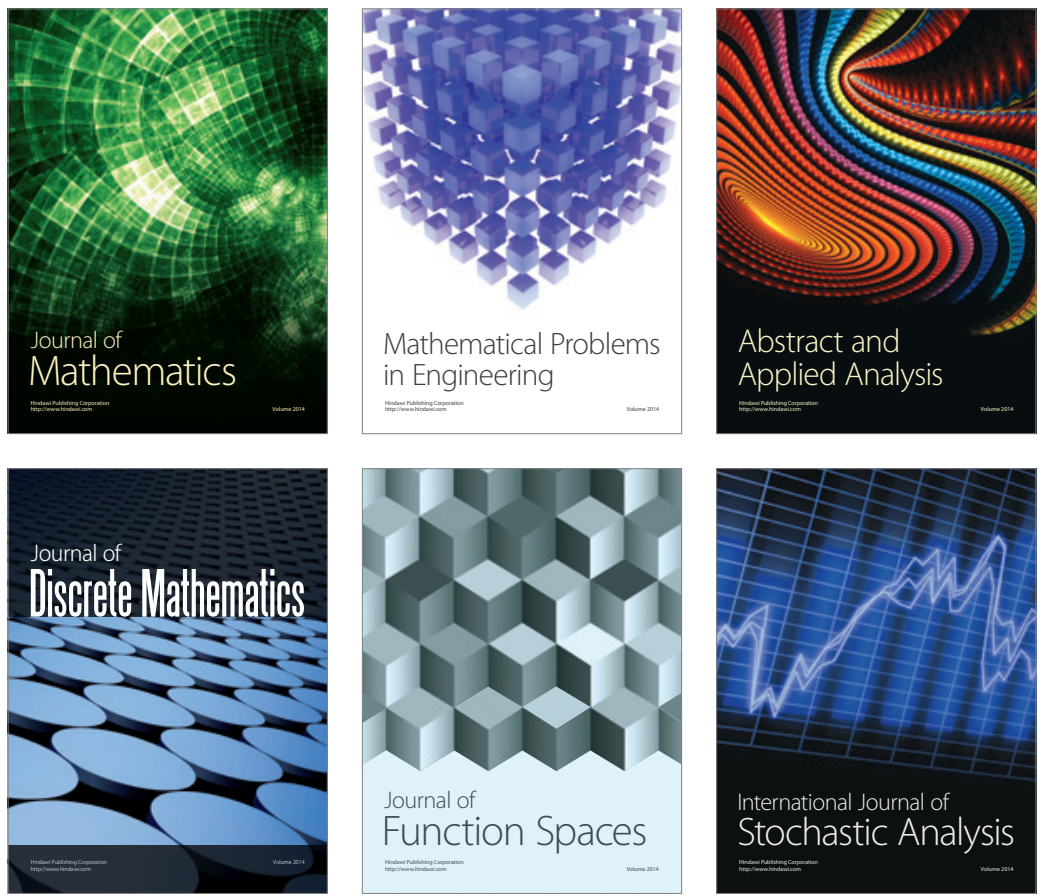

Journal of

Function Spaces

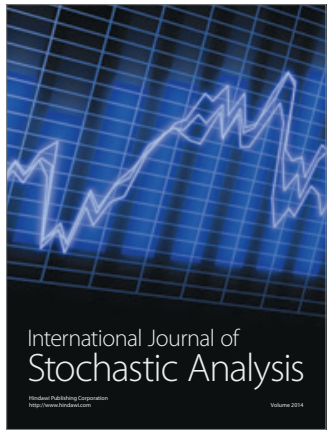

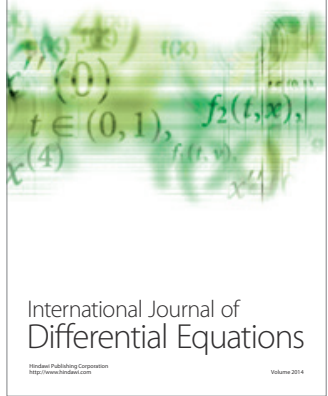
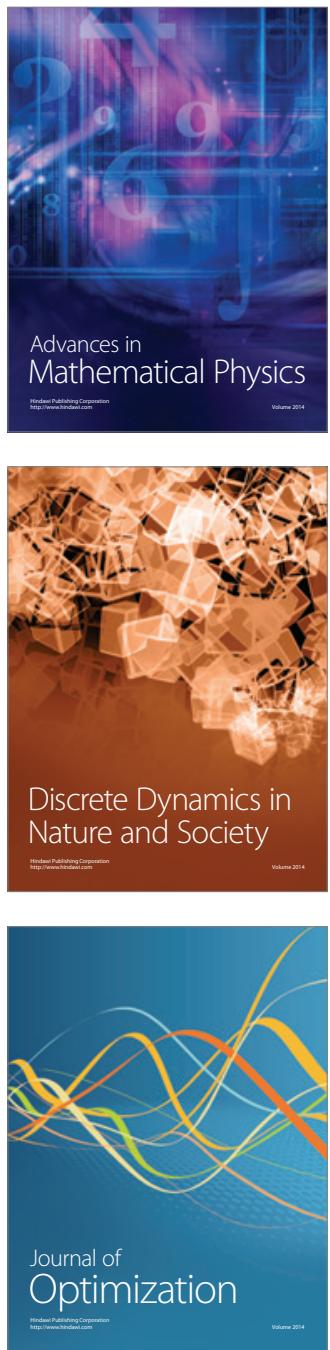